Dieter Senghaas, Zum irdischen Frieden. Erkenntnisse und Vermutungen, Frankfurt (Suhrkamp Verlag) 2004.

Mit Zum irdischen Frieden ist Dieter Senghaas, Leiter des Instituts für Interkulturelle und Internationale Studien in Bremen, ein in vieler Hinsicht konkludenter Forschungsbeitrag zur friedenspolitischen Debatte gelungen.

Obwohl kein weiterer »Beitrag zur Kant-Philologie«, knüpft der »irdische Friede « an die europäische Friedenstradition der Aufklärung an (S. 8). Auch Kants "Ewiger Friede« war ja nicht als ein endgültiger Dauerzustand gedacht, sondern irdisch: etwas durch ständiges Bemühen perpetuierlich Anzustrebendes, das nach Kant »selbst für ein Volk von Teufeln« erreichbar sei (»wenn sie nur Verstand haben«). Insofern bietet Zum irdischen Frieden zunächst nichts aufregend Neues. Doch Senghaas‘ Ausführungen gehen notwendigerweise weiter. Der Autor will »jene Erkenntnisse, Einsichten und auch Vermutungen, insbesondere aber wißbares weltkundiges Wissen, aufbereiten, die für ein zeitgemäßes differenziertes Verständnis der gegenwärtigen und absehbar zukünftigen weltweiten Friedensproblematik von Bedeutung sind «(S. 11). Dabei geht der Autor von vier »Welten « aus, auf welche moderne Entwicklungen wie z.B. Globalisierung auf verschiedene Weise Einfluss ausüben. Maßstab für Senghaas' Analyse ist das bekannte, in früheren Schriften bereits vorgestellte »zivilisatorische Hexagon«.

Senghaas bekennt sich zu einer konstruktiven Friedensursachenforschung. Konsequent bezieht der Autor dagegen Stellung, dass die Staaten immer noch »ein militärisch abgesichertes Machtmanagement « inszenieren (»para bellum-Maxime«), das wahrscheinlich »seinerseits die Eskalationsträchtigkeit von Gewaltlagen verschärft, anstatt potentieller Eskalation entgegenzuwirken und sie einzudämmen «. Senghaas kritisiert, dass »unter den Vorzeichen des militärischen Primats ... die Instrumentarien der friedlichen Streitbeilegung und selbst noch relativ militärnahe Instrumentarien wie ... kollektive Sicherheit « als sekundär eingestuft werden (S. 17f.).

Erforderlich sei, die para bellum-Maxime in die »kontrastierende Maxime« si vis pacem, para pacem aufzulösen. Ein »zeitgemäßes Friedenskonzept « sei demnach die universale »Herrschaft des Rechts « (rule of law), die jedoch einer »innergesellschaftlichen und internationalen Friedensstruktur « bedarf. Zwischen beiden »bestehen Rückkoppelungen mit wechselseitigen Verstärkungseffekten $«(S .23)$.

Senghaas rekurriert auf den »ursächlichen Pazifismus « (nach dem Friedensnobelpreisträger Alfred H. Fried), der darauf aus ist, die Ursachen für Kriege zu beseitigen. Nach Fried: »...wer eine neue wünschbare Folge anstelle einer anderen gezeitigt sehen will, muss anstelle der einen Ursache jene andere setzen, die die gewünschte Folge hervorbringen kann « (Senghaas, S. 26). Eben dies war Gegenstand der Haager Friedenskonferenzen 1899 und 1907, welche den Krieg abschaffen und an dessen Stelle den Rechtsweg verbindlich vorschreiben wollten; nur so, so meinte man, würde $\mathrm{Ab}$ rüstung überhaupt möglich. Diese »klassische Lehre« des Pazifismus bezweckte eine »neue Weltordnung«, nicht die »Beseitigung von Konflikten «. sondern die »Konflikttransformation « (S. 27), nach Fried: »Die Umwandlung des zwischenstaatlichen Verhältnisses, ... [so] daß sie [die Konflikte] der Gewaltlösung entrückt und [der] rechtlichen Behandlung « zugeführt würden (Senghaas, S. 27). Für Senghaas steht dabei eine "soziale Ordnung « im Vordergrund, die sicherstellt, dass »Konflikte in aller Regel verläßlich gewaltfrei bearbeitet werden, also im politischen Sinne des Begriffs Frieden hergestellt ist « (S. 26).

Auf den Seiten 26 bis 80 folgt eine Aufarbeitung des Diskurses, der bereits im Zusammenhang mit den Haager Konferenzen stattgefunden hatte, allerdings nun unter Einbeziehung neuer aktueller, ganzheitlicher sozialer und politischer Sachverhalte. Obwohl damals schon ein $»$ wissenschaftliches wie praktisches Anliegen «, wich die Vorstellung einer auf das Recht gegründeten Organisation des Friedens im 20. Jahrhundert (dem Jahrhundert »der Gewaltherrschaft, der Kriege, der Genozide, der wechselseitigen Vernichtungsdrohung «) einem z.T. oberflächlichen »Antimilitarismus «.

So konnte die »Lücke« - die (fehlende) internationale Rechtsordnung - im 20. Jahrhundert nicht gefüllt und der »Aufbau dauerhafter friedensfördernder Strukturen und Mentalitäten « nicht erreicht werden (S.27). Inzwischen sind, wie der heutige $»$ Blick in die Welt zeigt, ... Staatsverfall und eine Fülle von militanten innerstaatlichen Konflikten ... zu beobachten« (S. 28). In diesem Zusammenhang wäre ein Hinweis hilfreich gewesen, warum die dafür ursächlich verantwortlichen europäischen Mächte ihren
Anspruch, in den internationalen Beziehungen den Primat des Rechts vor dem der Macht durchzusetzen, noch immer nicht realisiert haben. Eine genauere Analyse der bereits zitierten »Rückkoppe-

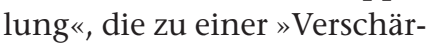
fung der Eskalationsträchtigkeit « führt und geführt hat, könnte aufklärend sein. Vielleicht eignet sich Senghaas‘ Anwendung der »Grundstruktur des Freudschen Modells ... auf die Analyse des Verhaltens von Staaten und Nationen « auch als Apologie der nördlichen Industrieländer (S. 111). Ähnlich wie beim Freudschen »Ich « spielen bei modernen Verfassungsstaaten Abwehrmechanismen, Verdrängung, Projektionen, Verneinung, Todestrieb eine Rolle - was geradezu »autistische Milieus « hervorbringt (S. 105). Staaten handelten generell, wie Senghaas richtig unterstellt, »viel weniger rational, als es die Theorie behauptet $«($ S. 82).

Gerade angesichts eines solchen pathologischen Befundes ist jedoch fraglich, ob es notwendig ist, dass »sich in einem bisher eher konfliktabgeneigten Kulturbereich (wie dem konfuzianisch-buddhistischen) eine politische Kultur verankern kann [bzw. soll], die zu Konflikten und Konfliktverarbeitung allmählich ein positives Verhältnis gewinnen wird «(S. 44). Argumentiert der Autor hier gegen das in diesen Kulturen zur Lösung von Konflikten gebräuchliche Konsensprinzip?

Der anarchische Zustand muss auf jeden Fall beendet werden, damit, so Senghaas, der »aus politisierter Differenz resultierende, moderne soziale Konflikt' ... mit [seinen] erheblichen externen Folgen « nicht - statt zu wünschenswerter Koexistenz - zur »Fundamentalpolitisierung « 
(S. 30) und statt zu einer »Mäßigung des Konfliktverhaltens ... einer Zähmung der Affekte, ... Aggressionshemmung und Gewaltverzicht, ...[und] darauf aufbauend Toleranz und Kompromissfähigkeit ...[sowie] geregelter Rechtsfortbildung «(S. 34) zu Terrorismus, Apathie oder einer »Libanisierung « der Verhältnisse führt. Es muss also, da »die Fundamentalpolitisierung der Welt voranschreitet ... [z]ivilisierte Konfliktbearbeitung ... dem zu bewältigenden komplexen Problemknäuel gewachsen sein « (S. 137).

Hinsichtlich der Verteilungsgerechtigkeit in »sich modernisierenden Gesellschaften « (S. 157) stellt sich die Frage, wie es innerhalb von und zwischen den armen Staaten der südlichen Erdhalbkugel Gerechtigkeit und konstruktive Konfliktbearbeitung geben kann, wenn auf internationaler Ebene wesentliche Voraussetzungen dafür fehlen. Senghaas schlägt zwar nicht vor, statt Waffen- und Entwicklungshilfe für schwache Staaten zu leisten, sollte man ihnen für die von ihnen gelieferten Rohstoffe und Bodenschätze reelle Preise zahlen, die Entwicklungshilfe überflüssig machen würden. Der Autor betont jedoch die Notwendigkeit konstruktiver Beiträge sowohl im Hinblick auf eine »weltweite sschicksalsgemeinschaft « und die »Gesellschaftswelt « und »Wirtschaftswelt « dieser Regionen, welche asymmetrische Interdependenzen abbauen helfen würden.

Dass Senghaas kein Asienwissenschaftler ist, wird deutlich, wenn er »im ethnopolitisch zerklüfteten Indien « zu beobachten meint, dass »der postkolonial-säkulare Staat unter dem Beschuss fundamentalistisch(er) ... Kräfte« zusammenbrechen könnte und die Menschen »demnächst « vor der Entscheidung stünden, »den säkularen Staat als institutionelle Plattform einigermaßen zivilisierter Konfliktbearbeitung « abzuschaffen und, »ihn ersetzend, eine neue, die Ethnokonflikte einhegende Plattform zu (er)finden « (S. 46f.). Es erübrigt sich hier ins Detail zu gehen. Trotz augenfälliger Unterschiede: Immerhin hat Indien als größte Demokratie der Welt bereits erreicht, wonach Europa noch strebt: die politische Einheit und eine demokratische föderale Verfassung. Freilich: Weder in Indien, noch in Europa waren diese Entwicklungen ohne Hilfe von außen möglich.

Auch Gandhis »traditionsbewußte Lebensphilosophie « war nicht nur »dörflich, antikommerziell und egalitär ausgerichtet « und sah nicht nur »kleine Einheiten ... im überschaubaren Raum " vor (S. 49). Gandhi und mit ihm die gesamte Kongresspartei befürworteten eine föderale Weltordnung (world federation), eine Art erweitertes Commonwealth, in dem Indien eine ihm angemessene Rolle spielen sollte.

Was also Europa »auf Wegen, Umwegen und auch Abwegen lernen musste, ... wird sich als Vorgang in anderen Teilen der Welt ... wiederholen müssen: Die nicht aufschiebbare Bewältigung der Koexistenzproblematik angesichts um sich greifender Fundamentalpolitisierung in der Folge des zitierten Umbaus traditionaler in sozial moderne Gesellschaften...«(S. 47f.). Nach Senghaas beinhaltet dieser Satz keine Bevormundung, da in Europa selbst diese Entwicklung nicht »vorgesehen « war und es anfällig bleibt für Fehl- und Rückentwicklungen. Allerdings erfüllt Europa das zivilisatorische $\mathrm{He}$ - xagon: »Toleranz, Sensibilität für Spielregeln, Mäßigung, Gewaltenteilung, Kompromissbereitschaft, ... Sinn für mehr als das eigene Interesse (Empathie) « am ehesten; diese Eigenschaften sind »Ergebnisse von mühsamen kollektiven Lernprozessen «. Um den Vorwurf des Eurozentrismus abzuwehren, versichert der Autor, »der demokratische Verfassungsstaat [sei] nicht das Ergebnis kulturgenetischer Vorprägung «(S. 39). Fraglich ist allerdings, ob ein Europa, das weiterhin auf militärische Friedenssicherung setzt und eine umfangreiche Rüstungsexportpolitik betreibt, als eine "Zone des Friedens « gelten kann, in der »der Krieg ... abgeschafft « ist (S. 163). Auch scheint in entscheidenden Fragen der kollektive Lernprozess noch nicht abgeschlossen.

Senghaas kontrastiert ferner die der europäischen Geschichte innewohnende Dynamik mit der Statik traditioneller Gesellschaften, in denen »die Rollen und Rollenspiele der Handelnden vorgezeichnet (waren). Zyklizität bestimmte das historische Selbstverständnis, das in Wirklichkeit kein im heutigen Sinne historisches war, weil der Zyklus - analog zu den Vorgängen im Jahresrhythmus der Natur ... immer wieder zum selben Ausgangspunkt zurückkehrte« (S. 48). Hier irrt der Autor, denn zwar ist das Geschichtsverständnis in asiatischen Ländern zyklisch, aber ebenso wie in der Natur kann es niemals eine Rückkehr exakt zum selben Ausgangspunkt geben: Die Bewegung ähnelt eher einer Spirale - wie ja auch Modelle aus der Biologie nahelegen. Steht die Menschheit insgesamt gegenwärtig vor dem Hintergrund von Globalisierung und »Fundamentalpo- litisierung « im Begriff, eine neue Evolutions- beziehungsweise Organisationsstufe zu erklimmen? Senghaas scheint dieser Idee nicht abgeneigt.

Trotz der hier geäußerten Kritik ist das Buch von Dieter Senghaas immens lesenswert und enthält zahlreiche wohlgemeinte, anschauliche und auch praktische Vorschläge, wie eine »politische Kultur konstruktiver Konfliktbearbeitung «(S. 157) geschaffen werden kann.

Klaus Schlichtmann

Peter Waldmann, Terrorismus: Provokation der Macht. 2. vollst. überarbeitete Ausgabe, Hamburg (Murmann Verlag) 2005.

Die zweite Ausgabe des Buches von Peter Waldmann erfuhr im Vergleich zur 1998 erschienenen ersten Ausgabe (rezensiert in S+F 2/2000, S. 197f.) eine deutliche Aktualisierung. Vormals fehlenden Bezügen zu modernen Erscheinungsformen des Terrorismus in den 1990ern, insbesondere zum religiös motivierten Terrorismus, wird im vorliegenden Buch nun Rechnung getragen. Nichtsdestotrotz weiß sich Waldmann in seiner sachlichen und gut verständlichen Analyse nicht ausschließlich auf die neuen terroristischen Organisationen zu beschränken, sondern ein durchaus nachvollziehbares und umfassendes Bild des Terrorismus zu zeichnen. Aspekte modernen Terrorismus werden dabei in Verbindung mit historischen Entwicklungen und sozialpsychologischen Fragen betrachtet. Der sozialwissenschaftliche Blickwinkel richtet sich somit nicht nur auf 
terroristische Organisationen und ihre eher technischen Seiten, sondern auch auf menschliche und gesellschaftliche Begebenheiten. Der Autor tut dabei gut daran, keine persönlichen Wertungen einzubringen und objektiv an seinen Untersuchungsgegenstand heranzugehen. Es geht ihm insgesamt darum, Terrorismus als soziales Handeln zu verstehen und in der Folge zu erklären und zu prognostizieren. Er verzichtet dabei auf moralische oder rechtliche Beurteilungen, wie es anderen Disziplinen, die sich mit Terrorismus auseinandersetzen, oftmals anheim ist. Das ist ihm in der Tat gelungen, was dem Leser sicherlich ein im Sinne Waldmanns differenziertes Bild des Terrorismus beschert.

Waldmann stellt eingangs eine Definition des Terrorismus auf, die er in den folgenden zwei Kapiteln erläutert: »Unter Terrorismus sind planmäßig vorbereitete, schockierende Gewaltanschläge gegen eine politische Ordnung aus dem Untergrund zu verstehen. Sie sollen vor allem Unsicherheit und Schrecken verbreiten, daneben aber auch Sympathie und Unterstützungsbereitschaft erzeugen «(S.12). Konsequent kommt Waldmann im Laufe seines Buches immer wieder auf die einzelnen Punkte seiner Definition zurück, was das Verständnis erleichtert. Wie Waldmann beklagt, scheitert ein sachlicher Umgang mit dem Problem des Terrorismus in der politischen Wirklichkeit gerade schon an einer fehlenden einheitlichen Definition. Die genannten Merkmale Planmäßigkeit, Öffentlichkeit und politische Motivation, aus dem Untergrund, Schockeffekt und Sympathieerzeugung - führen schließlich zum Kern der Definition: Terrorismus »ist primär eine Kommunikationsstrategie (S.15). Das heißt, Terrorismus habe als extreme Variante asymmetrischer Kriegsführung (denn Terroristen haben angesichts der staatlichen Übermacht, gegen die sie als kleine und schwache Gewaltverbände kämpfen, keine Möglichkeit, eine offene Konfrontation auszutragen) vor allem einen symbolischen Stellenwert. Terrorismus, sozusagen als »Verlegenheitsstrategie « (S.13), solle sowohl Aufmerksamkeit und Sympathie (ein Punkt, den Waldmann im Vergleich zu anderen Autoren besonders hervorhebt) für eine Sache herstellen, als auch den Gegner zu überzogenen Reaktionen verleiten, um ihn in letzter Konsequenz selbst als Aggressor auftreten zu lassen. Zur umfassenden Definition von Terrorismus gehöre auch eine Abgrenzung von den Nachbarbegriffen Terror, Guerilla und gewöhnliche Kriminalität. So gebe es zwar Vermischung und Kombinationen dieser öffentlich wirksamen Gewaltformen, es lägen ihnen jedoch unterschiedliche Handlungslogiken zugrunde.

Waldmann nimmt noch weitere Unterteilungen vor. Unter Verwendung geeigneter Grafiken verdeutlicht der Autor die Entwicklung des internationalen Terrorismus sowie seine Abgrenzung zum nationalen Terrorismus. Beachtenswert sei vor allem, dass, entgegen öffentlicher Perzeption, nur etwa 15 Prozent aller terroristischen Anschläge als international klassifiziert werden können. Ferner geht Waldmann aus aktuellem Anlass auf die Gegenüberstellung von »altem « und »neuem « Terrorismus ein. Einleuchtend argumentiert er, dass sich in den letzten Jahren zwar einzelne
Strukturen des Terrorismus gewandelt hätten, dies jedoch keineswegs als ein »neuer « Terrorismus bezeichnet werden könne. Wenngleich insbesondere die Al Quaida als erste von vornherein transnational angelegte Terrororganisation einen deutlichen »Innovationsschub « darstelle, kann Waldmann zeigen, dass als neu dargestellte Erscheinungen wie dezentrale Organisationsstruktur oder religiöse Motivation mit Parallelen aus der Geschichte des Terrorismus widerlegt werden können. Die Grundfragen des Terrorismus blieben bestehen.

Das terroristische Kalkül selbst entspreche einem rationalen Zweck-Mittel-Denken. Es sei ein kompliziertes und indirektes Kalkül, das über Zwischenstufen zum Erfolg führen solle. Die Überlegung beruhe dabei auf der Idee der Provokation: Gemäß einem »Provokations-Repressions-Schema « müssten die Gewaltbotschaften erstens wahrgenommen und verstanden werden, und zweitens der Adressat der Gewalt im Sinne der Terroristen überreagieren, um somit die Masse der Sympathisanten zur offenen Rebellion zu bewegen. In der angestrebten Reaktion wiederum liege aber auch die immanente Schwachstelle des terroristischen Kalküls, da Terroristen lediglich einen Anstoß geben könnten, ohne ihre Zielerreichung weitergehend $\mathrm{zu}$ beeinflussen. Weil die Reaktionen schlecht vorhersehbar seien, falle die Erfolgsbilanz auf die Ziele der Terroristen bezogen nicht gut aus. Lediglich hinsichtlich des destruktiven »Störens « durch Anschläge könne man Erfolg feststellen. Dieser stelle sich jedoch nicht positiv dar, sondern nur negativ.
Im Folgenden geht Waldmann auf die geschichtlichen Vorläufer des Terrorismus ein, denn dieser sei keine Erfindung der Neuzeit. Trotz historischer Präzedenzfälle sei Terrorismus als Gewaltstrategie eigener Art aber erst mit der Schlussphase der Französischen Revolution bekannt geworden. Detaillierter widmet er sich dem 19. und 20. Jahrhundert, indem er einige Terrorgruppen und -praktiken beschreibt. Ausgehend von diesen vorbereitenden Entwicklungen zieht Waldmann den Bogen zum modernen Terrorismus: »[...] vor allem ein schwacher, mit sozialen Integrationsproblemen und Legitimierungsschwierigkeiten kämpfender Staat, sich uferlos ausdehnende Megastädte und wachsende Gruppen unzufriedener Intellektueller, sind gegenwärtig weniger für das postmoderne Europa als vielmehr für die im Umbruch befindlichen Regionen der östlichen und südlichen Hemisphäre, beispielsweise den gesamten islamisch geprägten Ländergürtel, kennzeichnend. Insofern ist in absehbarer Zeit schwerlich mit einer Drosselung des Terrorismus - allein oder in Verbindung mit anderen Gewaltformen -, sondern eher mit seiner Fortdauer zu rechnen, selbst wenn die Motive, aus denen er sich speist und die Formen, in denen er geführt wird, einem Wandel unterliegen mögen« (S. 61f.).

Ein Kapitel behandelt die Ressourcen terroristischer Gruppen. Waldmann erläutert hier Organisationsstrukturen, Finanzierung und technische Ausstattung von Terroristen. Die »Hauptressource« der Terroristen sei »Motivation «. Diesen Faktor sowie seine jeweilige ideologische Rechtfertigung zieht Waldmann deshalb später zur Unter- 
scheidung verschiedener Terrorismustypen heran.

Dem technischen Faktor »Medien « ist, da er ein so wichtiger Bestandteil der Kommunikationsstrategie Terrorismus sei, ein eigenes Kapitel gewidmet. Waldmann setzt sich hier kritisch mit dem ambivalenten Verhältnis zwischen Terrorismus und Medien auseinander. Anhand des Beispiels indirekter Medienkontrolle während des Nordirlandkonfliktes kann er zeigen, dass man sich vor einer »Diabolisierung « der Rolle der Medien hüten solle, wenngleich sie integraler Bestandteil des terroristischen Kalküls seien.

Eine Einteilung verschiedener terroristischer Varianten nimmt Waldmann gemäß ihrer jeweiligen Motivation vor, selbst wenn sie sich gleichsam in anderen Aspekten unterscheiden. Aber »... letztlich sind es vor allem bestimmte, mit großer Inbrunst und Energie vertretene und mit anderen geteilte Überzeugungen, welche die Entstehung dieser Gruppen und ihre teils äußerst zähe Überlebenskraft erklären« (S.99). Er unterscheidet vier Kategorien: sozialrevolutionärer, ethnisch-nationalistischer, rechtsradikaler und religiös motivierter Terrorismus. Mit Beispielen wie der RAF und ETA zeigt Waldmann die unterschiedlichen Ausgangsbedingungen, Zielprogramme, Strategien und soziale Herkunft auf. Für Terrorismus prädestinierte Persönlichkeiten gebe es nicht. Es wird deutlich, dass Terroristen erstens keineswegs "Irre « seien, sondern durchaus rational vorgingen, und zweitens nicht Armut und Einfältigkeit Menschen in die Arme des Terrorismus führten, sondern man die meisten Terroristen als Intellektuelle bezeichnen könne. Dies kann Waldmann durch ein zusätzliches Kapitel unterstreichen, in dem er Lebensläufe unterschiedlich orientierter Terroristen beschreibt.

Der religiöse Terrorismus wird in der zweiten Ausgabe des Buches ausführlicher behandelt, insbesondere der islamistische Terrorismus und die Al Quaida. Dabei beschreibt Waldmann sowohl historische Beispiele religiösen Terrorismus, als auch die Renaissance des Islam im Speziellen. Er warnt dabei vor vorschnellen Einteilungen hinsichtlich der Gewaltbereitschaft der Religionen, denn allen sei Gewaltpotential inhärent.

Waldmann wendet sich gegen Walter Laqueurs Annahme, Terrorismus sage nichts über die jeweiligen Sozialstrukturen der Gesellschaft aus, in der er stattfinde. Er zeigt auf, dass sehr wohl ein Zusammenhang zwischen breiten Protestbewegungen in der Gesellschaft und Terrorismus besteht. Außerdem habe das politische System einer Gesellschaft Auswirkungen auf das Entstehen terroristischer Gruppen. Demokratien etwa seien viel anfälliger für terroristische Anschläge als Diktaturen.

Das vorletzte Kapitel behandelt die Eskalationsschraube von Isolierung und Radikalisierung von Terroristen. Hier kommt abermals die sozialwissenschaftliche Blickweise von Waldmann zum Tragen. Nachdem bisher vom rational agierenden Terroristen gesprochen wurde, setzt sich Waldmann nun mit der irrationalen Komponente terroristischer Gewalt auseinander, insbesondere mit dem Militarisierungsprozess, d.h. der »Verselbständigung der Gewalt«. Daneben geht er auf die unterschiedlichen Möglichkeiten der Beendigung terroristischer »Feldzüge « ein, ebenso wie auf die Wirkungen von Terrorismus auf die jeweils betroffenen Gesellschaften, deren politische Kultur und Landschaft meist negativ beeinflusst würden.

Waldmann schließt mit einem Kapitel zu Gegenmaßnahmen. Er möchte Möglichkeiten und Grenzen der Kontrolle des Terrorismus aufzeigen. Dabei geht er angenehm nüchtern und realistisch vor. Er plädiert vor allem für eine differenzierte Auseinandersetzung mit dem Problem. Dies bedeute einerseits, sich ausgiebiger mit den unterschiedlichen Strukturtypen des Terrorismus auseinander zu setzen. Andererseits müsse man sich realistischerweise klar machen, dass man Terrorismus niemals gänzlich ausmerzen könne. Wichtig sei, Terrorismus nicht zu dämonisieren, sondern ihn als pragmatisches Problem zu betrachten. Mit diesem Eindruck hinterlässt die Lektüre dieses sehr empfehlenswerten Buches auch den Leser.

Florian Roel

Martin H. W. Möllers/Robert Chr. van Ooyen (Hrsg.), Europäisierung und Internationalisierung der Polizei, Frankfurt (Verlag für Polizeiwissenschaft Dr. Clemens Lorei) 2006.

Die immer weiter fortschreitende europäische Integration und die zunehmende Vertiefung der Zusammenarbeit auch in den sensiblen Bereichen der »high politics« wie der Polizeilich- justiziellen Zusammenarbeit in
Strafsachen haben seit den 1990er Jahren im verstärkten Maße zu einer Europäisierung der Polizei geführt. Der »Raum der Freiheit, der Sicherheit und des Rechts « nimmt somit zunehmend Gestalt an. Dass diese Entwicklungen allerdings oft auch an ihre (nationalstaatlichen) Grenzen stoßen, hat nicht zuletzt die Zurückweisung des Europäischen Haftbefehls durch das Bundesverfassungsgericht gezeigt. Auch auf der globalen Ebene ist es durch neue Herausforderungen wie der des transnationalen Terrorismus sowie der failed und failing states und der damit verbunden Ausweitung des Sicherheitsbegriffs und der Neudefinition des Sicherheitsdilemmas zu einer verstärkten Internationalisierung der Polizei gekommen.

Diesen beiden Phänomenen - sowohl der Europäisierung als auch der Internationalisierung der Polizei - nehmen sich die Herausgeber Martin H.W. Möllers und Robert Chr. van Ooyen, beide Dozenten für Gesellschaftswissenschaften am Fachbereich der Bundespolizei der Fachhochschule des Bundes für öffentliche Verwaltung in Lübeck, in ihrem 21 Aufsätze umfassenden Sammelband an. Hierfür haben sie eine Reihe von zumeist schon an anderer Stelle veröffentlichten Beiträgen zusammen getragen. $\mathrm{Zu}$ loben ist an dieser Zusammenstellung vor allem die Auswahl unterschiedlicher Autoren: So finden sich neben Aufsätzen von Politikwissenschaftlern (Wilhelm Knelangen, Gert-Joachim Glaeßner, Astrid Lorenz, Thorsten Stodiek u.a.) und Juristen (Manfred Baldus, Anke Borsdorff u.a.) auch Beiträge von Autoren aus der Praxis (Manfred Eisele, Sven Jahn, Bernd Walter). Auch 
der innenpolitische Sprecher der SPD und Richter a.D. Dieter Wiefelspütz ist mit einem Beitrag zum »Einsatz der Bundespolizei im Ausland « in der bunten Autorenliste vertreten. Glänzt der Band also mit seiner breiten Auswahl an Autoren, kann man dies leider nur bedingt von seiner Aktualität behaupten. Dadurch, dass die Herausgeber auf meist schon veröffentlichte Texte zurückgegriffen haben, sind einige Beiträge eher älteren Datums. So zum Beispiel der von Möllers und van Ooyen verfasste Aufsatz über »Demokratie und Polizei« (S. 81-87) aus dem Jahre 2000 (!). Dies bedeutet, dass die einzelnen Aufsätze für sich genommen nicht neu sind, sondern lediglich ihre durch die Herausgeber vorgenommene Zusammenführung und Einordnung in den Kontext der Europäisierung bzw. Internationalisierung. Da aber in den meisten Fällen versucht wurde bei den entsprechenden Themen die aktuellen Entwicklungen (Scheitern des VVE, BVerfGEntscheidung zum Europäischen Haftbefehl) zu berücksichtigen und die Texte meist aktualisiert wurden, kann man über dieses Manko hinwegsehen.

Inhaltlich gliedert sich das Buch - dem Titel logisch folgend - in zwei Teile: Europäisierung (180 Seiten) und Internationalisierung (137 Seiten) der Polizei. Im ersten Teil werden unter anderem so vielfältige Themen wie die Europäische Grenzschutzagentur (Sven Jahn), die Entscheidung des Bundesverfassungsgerichts zum EUHaftbefehl (Robert Chr. van Ooyen) und das Schengener Einreise- und Visa-System behandelt (Tilmann Schott). Besonders interessant sind hier die Beiträge von Wilhelm
Knelangen, Dozent an der Universität Kiel, und Wolfgang Wagner, wissenschaftlicher Mitarbeiter an der Hessischen Stiftung Friedens- und Konfliktforschung. Knelangen untersucht in seinem Aufsatz über die »Europäische Union und die Bekämpfung des Terrorismus« den Einfluss des 11. September 2001 auf die Europäisierung der Polizei. Dabei kommt er schon in seiner Einleitung zu dem Schluss, dass die Anschläge in New York und Washington »alles andere als eine ,Stunde Null' für die politische Gestaltung der Rahmenbedingungen für die Zusammenarbeit von Polizeien, Strafverfolgungsbehörden und Geheimdiensten « (S. 70) gewesen seien. Trotzdem müsse der 11. September als »Wendepunkt « (S. 70) betrachtet werden, da viele der schon vorher in den neunziger Jahren ins Auge gefassten Pläne nun unter der Prämisse der Bekämpfung des Terrorismus durchgesetzt wurden. Knelangen stellt dar, dass die beschlossenen Maßnahmen oftmals gar keinen unmittelbaren Bezug zur Terrorismusbekämpfung aufwiesen, sondern »ursprünglich eine andere Hauptstoßrichtung - vor allem die Verfolgung schwerer und organisierten Kriminalität - hatten, vom Europäischen Rat und vom Rat der Innen- und Justizminister jedoch nunmehr als elementare Bausteine für die Bekämpfung von Terrorismus deklariert wurden « (S. 71f.).

In seiner Bilanz kommt Knelangen zu einem pessimistischen Schluss: Die Aufgabe der inneren Sicherheit liege weiterhin in den Händen der Mitgliedstaaten und bleibe durch nationale Regelungen geprägt (S. 78) - daran habe auch der 11. September nichts geändert. Für die Europäisie- rung der Polizei bedeute dies: »Trotz der gelegentlichen Vorschläge aus dem politischen Raum ist vor diesem Hintergrund die Schaffung einer zentralen europäischen Polizei, einer europäischen Staatsanwaltschaft oder eines europäischen Geheimdienstes nicht in Sicht $«$ (S. 78).

Ein ganz anderes Feld untersucht der Aufsatz von Wolfgang Wagner (»Polizei unter Kontrolle?«), der die Schwierigkeiten der parlamentarischen und gerichtlichen Kontrolle Europols darstellt. Dieses Problem ist besonders elementar, da bereits der Austausch von Daten - eines der Hauptarbeitsfelder Europols - einen Eingriff in die Grundrechte darstellt. Wagner verdeutlicht das Problem an einem Beispiel: »Während ... Fehler bei der Speicherung und Auswertung von Kundendaten durch Geschäfte schlimmstenfalls zu unwillkommenen Werbesendungen führen, können Fehler bei der Verarbeitung personenbezogener Daten durch Europol im schlechtesten Fall zu Verhaftungen, Wohnungsdurchsuchungen u.ä. führen « (104). Allerdings erscheinen Wagner nicht die datenrechtlichen Fragen als Hauptproblem, sondern vielmehr die Frage nach der »Kontrolle der Kontrolleure« (S. 100). Dem Konzept Andrew Moravcsiks folgend, demzufolge Regierungen durch Kooperation innenpolitische Themen $\mathrm{zu}$ außenpolitischen machen und sich somit der Kontrollinstanz der innerstaatlichen Akteure zu einem gewissen Grad entziehen können, stellt Wagner für die polizeiliche Kooperation ein besonderes Kontrolldefizit fest: Die nationalen Parlamente können ihre Kompetenzen nicht mehr ausüben, und das Europäische Parlament kann dies beim jetzigen Integrationsstand noch nicht (S. 101). Unter diesen Gesichtspunkten hält Wagner die Ablehnung des Verfassungsvertrags in Frankreich und in den Niederlanden für »sehr bedauerlich«, da er seiner Meinung nach einen deutlich besseren Grundrechtsschutz und eine verstärkte parlamentarische Kontrolle erbracht hätte (S. 110). Daher fordert Wagner in seinem Schlussplädoyer, diese Fortschritte als »kleine Vertragsänderungen « (S. 110) auch ohne Verfassungsvertrag umzusetzen.

Der zweite Teil (Internationalisierung) enthält ebenfalls eine Vielzahl unterschiedlichster Titel: So werden Themen wie Policekeeping (Manfred Eisele), der Aufbau des Grenzschutzes in Bosnien und Herzegowina im Auftrag der VN (Christian Mainzinger) und das Luftsicherheitsgesetz (Elmar M. Giemella) behandelt.

Der Beitrag von Thorsten Stodiek über den Aufbau von multi-ethnischen demokratischen Polizeien auf dem Balkan beruht auf Feldforschungen im Rahmen eines Forschungsprojekts am Zentrum für OSZE-Forschung (CORE) des Instituts für Friedensforschung und Sicherheitspolitik an der Universität Hamburg (IFSH). Somit kann der Autor interessante Einblicke in die Probleme des Aufbaus, der Verwaltung und der Struktur dieser Polizeieinheiten geben. Das Untersuchungsdesign umfasst die - vor allem durch die OSZE durchgeführten - Einsätze zum Aufbau multiethischer Polizeien im Kosovo, in Südserbien und in Mazedonien. Wie Stodiek darstellt, unterscheiden sich diese Mandate betreffend des möglichen Einflusses der internationalen Akteure massiv. So 
ist im Kosovo, dessen politisch-administrative Leitung dem UN-Übergangsverwalter obliegt, der Handlungsspielraum sehr groß: »Somit haben die UNO und die für den Aufbau mitverantwortliche OSZE freie Hand bei der Strukturierung des Polizeiapparats, der Entwicklung der Lehrpläne und der Sanktionierung der lokalen Polizei« (S. 227). In Südserbien und Mazedonien dagegen sei der Einfluss geringer, da die Aktionen auch von der Kooperationswilligkeit der einzelnen Regierungen abhängen. Der Erfolg der multi-ethnischen Einheiten war uneinheitlich. So konstatiert Stodiek, dass die gemischten Einheiten in allen Fällen bei der albanischen Bevölkerung einen »vertrauensbildenden Effekt « gehabt hätten (S. 227), die Serben und slawischen Mazedonier den multi-ethnischen Gruppen allerdings weiterhin skeptisch gegenüber stehen (S. 228). Auch der Ausbildungsstand der Polizeien sei aufgrund schlechter Schulbildung, zu kurzer Ausbildungsdauer und unzureichenden Lehrstoffkenntnissen problematisch. Darüber hinaus würden viele ungeeignete Bewerber - unter anderem aufgrund des Drucks von Rebellengruppen und politischen Parteien - in den Polizeidienst mitaufgenommen. Stodiek kommt zu dem Schluss: »Sollen die bisherigen Erfolge daher nachhaltig ausgebaut werden, müssen die internationalen Akteure weiterhin Ausbildungsprogramme für die neuen Polizeikräfte anbieten und die Innenministerien zu einer ernsthaften und nicht nur symbolischen Integration der multi-ethnischen Einheiten in die regulären Polizeistrukturen anhalten « (S. 230).
Insgesamt kann der Sammelband aufgrund seiner thematischen Breite und Tiefe überzeugen. Bedauerlich bleibt allerdings, dass - wie bereits dargelegt - wenig neues Material (auch wenn die meisten Artikel in einer modifizierten Form vorliegen) in dem Band $\mathrm{zu}$ finden ist. Ihrem selbst erklärten Ziel, Beiträge zu präsentieren, »die weite Teile eines längst nicht abgeschlossenen Prozesses in einer >Momentaufnahme hinreichend konturieren und die mit ihm verbunden aktuellen Schwierigkeiten deutlich beschreiben « (S. 8), werden die Herausgeber aber auf jeden Fall gerecht.

Jan-Hendrik Lauer

Arnold Bartetzky/Marina Dmitrieva/Stefan Troebst (Hrsg.), Neue Staaten - Neue Bilder? Visuelle Kultur im Dienst staatlicher Selbstdarstellung in Zentral- und Osteuropa seit 1918, Köln (Böhlau Verlag) 2005.

Dieser Sammelband verbindet in seinen rund 360 Seiten visuelle Kultur und Geschichtskultur zur visuellen Geschichtskultur. Er resultiert aus einem Projekt an der Universität Leipzig, welches darauf abzielte, staatliche Entwicklungen und deren Visualisierung in Ostmitteleuropa seit $1918 \mathrm{zu}$ untersuchen. Dabei werden die Umbrüche nach 1918, in der Zeit des Dritten Reiches, nach 1945 sowie in der Zeit von 1989 bis 1991 als Zeiten staatlicher Neuorientierung und damit auch Neuinszenierung betrachtet. Die deutsch- und englischsprachigen Beiträge sind von vielfältiger Natur und reichen von verschiedenen Instrumentalisierungen von Kunst und Architektur für politische Aussagen sowie zur Legitimation einer neuen Staatsmacht - durch Staatsapparate oder einzelne Personen - bis hin zur Konstruktion nationaler Identitäten, Reinterpretation von geschichtlichen Motiven, der Integration traditioneller Motive und moderner Technologie und schließlich strukturellen Analogien nach staatlichen Neuorientierungen. Hierbei wurden spezifische Bauwerke, Denkmäler und Museen wie auch Konzepte für ganze Städte, Bilder und Zeichnungen, Plakate, Geldscheine, Choreographien von Staatsfeierlichkeiten, aber auch Internetrepräsentationen miteinbezogen, veranschaulicht durch über 100 Illustrationen. Neben den Beiträgen über Zentral- und Osteuropa wurden auch zwei Beträge zur Türkei und Israel eingebracht. Der Sammelband assoziiert sich mit dem in den frühen 1990er Jahren entwickelten kulturwissenschaftlichen Ansatz des visual turn. Untersucht wird, wie Staaten Bilder wählen, um sich von vorangegangenen Zeiten abzugrenzen oder um geschichtliche Verbindungen zu betonen oder zu konstruieren - mit dem übergreifenden Ziel, den eigenen Anspruch auf Herrschaft zu legitimieren. Thematisch sind die Beitrage in fünf Abschnitte gegliedert: Neue Staaten nach 1918, Drittes Reich, Neue Staaten nach 1945, Neue Staaten nach 1989 und Brüche und Kontinuitäten: 1918-2003. Die Erwähnung jedes einzelnen Beitrags würde den hier gegebenen Rahmen sprengen, doch stellt jeder Beitrag ein wichtiges Stück im Gesamtwerk dar.

Im ersten und umfangreichsten Abschnitt - Neue
Staaten nach 1918 - wird zum Beispiel die Funktion der Hauptstadt in den Planungen des frühen 20. Jahrhunderts untersucht. Interessant hierbei ist das Resultat, dass Modelle von Gesamtplänen oder spezifischen städtebaulichen Elementen oftmals keine eigene Bedeutung hatten, sondern sich auch in unterschiedliche politische Richtungen einpassen ließen. Bei der politischen Selbstdarstellung finden sich ähnliche Elemente in den zentral- und osteuropäischen Staaten wie auch in anderen Ländern, neu sind diese nur mit Blick auf die jüngste Vergangenheit im jeweiligen Land. Ein anderer Beitrag zeigt am Beispiel der polnischen Hafenstadt Gdynia, wie Städte durch geschichtliche Entwicklungen und Einflüsse externer Mächte an landespolitischer Bedeutung gewinnen und verlieren. Zwei Beiträge zu Litauen beschreiben die Repräsentation von nationaler und kultureller Souveränität wie auch des Fortschritts in jungen Staaten, besonders durch architektonische Mittel. Im Beitrag zur Türkei wird gezeigt, wie emigrierte westliche Stadtplaner und Architekten die neue politische Kultur der kemalistischen Ideologie visualisieren und integrieren sollten: Ankara wurde so zum Ausdruck der Moderne. Dabei wird Religion in der Darstellung ganz bewusst ausgelassen. Ein Beitrag über Malerei illustriert, wie Bilder politische und geschichtliche Ideen zum Staat visualisieren. Durch die gezeigten Motive sagen Bilder etwas aus. Doch ist auch Verstecktes aussagekräftig - wobei hier etwas Verstecktes als eine oft unbewusste Aktion des Künstlers gesehen wird, aber dennoch motiviert durch geschichtliche Erfahrungen. Ein anderer Beitrag zeigt am Beispiel 
Rumäniens die historische Verankerung und Legitimation von Herrschaft durch zeremonielle und ikonographische Aspekte. Identitätsstiftung und -stärkung in Polen durch Medaillen und Abzeichen ist Thema eines weiteren Beitrags. Hier wurden politische Ereignisse und Themen visuell auf Medaillen verschiedener Ausführungen ausgedrückt und erlaubten so ein gemeinsames Identitätsgefühl in allen polnischen Bevölkerungsschichten. Die zum Teil des kollektiven Gedächtnisses gewordenen nationalen Motive glichen die fehlende Unabhängigkeit aus und trugen während des Ersten Weltkriegs zur Stärkung der polnischen Identität bei. Die zwei letzten Beiträge dieses Abschnitts beschäftigen sich mit staatlichen Versuchen, aus Individuen eine Gemeinschaft $\mathrm{zu}$ formen. Im ersten geht es um die ikonographische Umsetzung von Lenins Ideen für die Masse am Beispiel der Gestaltung von Stadträumen und staatlichen Festlichkeiten. Ideologisch kompatible Vorbilder wurden zur Übermittlung politischer Botschaften neu in Szene gesetzt, die auch in so genannten Agitzügen und Agitdampfern als Plattformen für Agitation ihren Ausdruck fanden. Traditionelle und sowjetische Symbole wurden verbunden. Wo anfangs ein erstaunliches Maß an Kreativität erlaubt war, gab es später feste Strukturen für den Sozialistischen Realismus. Im anderen Beitrag geht es um die pseudoreligiöse Qualität von Symbolen der Staatsmacht, die dadurch ihr Weiterleben sicherten. Um die Vergangenheit nicht nur zu verdrängen und ein weiteres Dasein dieser Symbole zu ermöglichen, wird vor ihrem bloßen Austausch oder Überstreichen gewarnt.
Im zweiten Abschnitt - Drittes Reich - wird die Gestaltung von Architektur und Raum zur Stärkung der staatlichen Macht behandelt. Im ersten Aufsatz geht es um die Monumentalität der staatlichen Architektur und um Architektur als suggestivem Inszenierungsmittel, um Stimmungsarchitektur. Auch wenn architektonisch Heldentum und Opfertod betont wurden, so weckte man doch statt Angst die Begeisterung der Massen. Der nächste Beitrag bespricht die Neugestaltung des in den Besitz der Nationalsozialisten gelangten Posen. Durch die Erfahrung der NS-Autorität mittels der Architektur sollten die Menschen nach Wunsch der Besatzer geformt werden. Bebauung und Neugestaltung sollten den Raum organisieren, unerwünschte Menschen verdrängen und die neuen Werte ausdrücken. Durch die Gestaltung von Wohnungseinrichtungen wurde auch das Privatleben miteinbezogen; somit war die Präsenz des Regimes allumfassend. Auch wenn Raumgestaltung auf das Formen menschlichen Verhaltens abzielte, war es schließlich doch nur ein Ausdruck der Besessenheit und Kontrolle. Der letzte Text dieses Abschnitts beschäftigt sich mit humoristischen Zeichnungen $\mathrm{zu}$ Albert Speers Neugestaltungsplänen für Berlin. Da einerseits die Überzeugtheit der Planer von ihrem Auftrag zum Ausdruck kommt, andererseits aber auch ein Bewusstsein über seine Absurditäten, geben diese Zeichnungen hinsichtlich der damaligen Politik Rätsel auf, die zugegebenermaßen auch für den Autor ungelöst bleiben.

Der dritte Abschnitt - Neue Staaten nach 1945 - beginnt mit einem Beitrag über den
Wiederaufbau von vier osteuropäischen Städten nach 1945 als politische und zentral organisierte Aufgabe. Der Grad der historischen Rekonstruktion hing auch von der politischen und geschichtlichen Wertung der Städte ab, wie am Kontrast zwischen Warschau und Gdansk illustriert wird. Warschau wurde als Heldenstadt gesehen und erfuhr darum eine gründlichere Rekonstruktion. Gdansk hingegen wurde auch mit der früheren deutschen und NS-Präsenz assoziiert, was zu einer oberflächlicheren historischen Rekonstruktion führte. Im Vergleich zum Wiederaufbau von Minsk und Kaliningrad zeigen die polnischen Beispiele einen größeren zentralen Willen, historische Städte zu rekonstruieren. Dies begründet sich auch darin, dass die neue polnische Regierung sich in stärkerem Maße legitimieren musste als die sowjetische, da letztere auch machtpolitisch weitaus besser etabliert war. Ein anderer Beitrag behandelt die Gestaltung des Knesset-Gebäudes in Jerusalem, des israelischen Parlaments, welches die Dauerhaftigkeit des Staates Israel visualisieren soll. Die architektonische und symbolische Verbindung des Parlamentsgebäudes mit der Gedenkstätte für die Opfer des Holocaust zielt auf die Verbindung von Vergangenheit und Zukunft. Eine steinerne Mauer und eiserne Tore umgeben das Plenum und drücken die kollektive Erinnerung aus. Biblische Zeiten und Holocaust dienen der Legitimitäts- und Identitätsstiftung. Doch gibt es auch vermehrt warnende Stimmen gegen die bewusst angewandte Identitätsstiftung durch den Holocaust - denn so würde sich Israel immer nur als Land der Opfer verstehen, unfähig, sich in anderen Kontexten auch als Täter zu sehen. Im letzten Beitrag dieses Abschnitts geht es um die Gedenkstätte in Treblinka und die Angemessenheit der Erinnerung an die Opfer des Holocaust. In Debatten über letzteres wuchs die Idee, dass der Holocaust ein Bruch der menschlichen Geschichte darstellt. Und so drückt auch die Gedenkstätte in Treblinka Zerrissenheit und Diskontinuität aus.

Der vierte Abschnitt - Neue Staaten nach 1989 - beginnt mit einer Darstellung eines gewissen politischen und gesellschaftlichen Vakuums nach dem Ende des Sozialismus und des Bedarfs einer neuen Identifizierung. Dabei spielten Denkmäler eine interessante, weil öffentliche Rolle. Viele wurden zerstört, andere wurden Exponate von Museen. Wiederum andere wurden in neue Zusammenhänge integriert. Durch ihre Behandlung drücken Denkmäler ihre unterschiedliche Bedeutung jenseits der Macht aus. Zerstörte Denkmäler repräsentierten ein Zuviel an staatlicher Macht. Denkmäler in Museen werden als historisch betrachtet. Denkmäler in neuer Form verloren ihre ursprüngliche Symbolik, und Denkmäler als Kaufware verloren den Ausdruck von Allmacht. Der nächste Aufsatz beschäftigt sich mit der Darstellung von Diktatur in heutigen Museen, was nicht immer objektiv geschieht. Zum Beispiel wird in den Ausstellungen ausgelassen, dass der Sozialismus auch ein Hoffnungsträger für die Menschen war. Ein weiterer Aufsatz behandelt das Internet als Mittel zur Verbreitung des Selbstverständnisses und der politischen Legitimation der Dnjestr-Republik. Es wird eine Geschichtspolitik betrieben, deren Erfolg sich mittler- 
weile in der Entwicklung der Idee eines transnistrischen Volkes zeigt.

Im fünften Abschnitt - Brüche und Kontinuitäten: 1918-2003 - wird unter anderem die Instrumentalisierung der Antike zur Stiftung einer nationalen Identität in Rumänien untersucht. Dabei wurde von den unterschiedlichen politischen Regimes einmal die Verbindung zu den Dakern, ein anderes mal die zu den Römern hervorgehoben. Während mit Antritt der Kommunisten jedoch die Slawen eine größere Bedeutung erfuhren, bezog sich die rumänische Regierung seit der Emanzipation von 1964 wieder auf die Antike. Die dakische und römische Vergangenheit wurde für die politische Sicherung des Ceaucescu-Regimes in- szeniert. Zwei weitere Beiträge beschäftigen sich mit Geldscheinen als Mittel zur Übertragung politischer Botschaften, zugunsten einer propagierten nationalen Identität, sowie zur Abgrenzung von anderen Staaten oder vergangenen Epochen. Die veränderte Staatsideologie ist besonders bildhaft am Beispiel Jugoslawiens dargestellt. Ein anderer Text analysiert die Bedeutung von Erde in der Erinnerungskultur. Erde wird oft mit Emotionen und politischen Ideen verbunden, wie eine Installation im nördlichen Lichthof des Reichstags in Berlin zeigt. Erde wird mythologisch als Ernährerin, anthropologisch als Heimat oder auch funktionalistisch als Mittel zur Herrschaftslegitimation, Identitätsstiftung und -erhaltung wie auch zum Gedenken gesehen. Als Grundmuster wird die Beschwörung positiver Zustände gesehen, auch wenn zum Verständnis der Bedeutung für den Einzelnen der Kontext hinzugezogen werden muss. Schließlich geht es im letzten Beitrag um die Darstellung des Staates mittels gymnastischer Massenaufführungen, die es dem Staat erlaubten, mehr als nur sich selbst zu zeigen. Im Vergleich zu den Aufführungen während der NS-Zeit kam in den kommunistischen Ländern jedoch auch zum Ausdruck, wie sich ihre Gesellschaften selbst feierten.

Der Band resümiert, dass die gewählten Formen allein keine ideologische Bedeutung besitzen, sondern dass diese erst durch die Art der Verwendung oder Darstellung gewollt hinzugefügt wird. Es gelingt ihm, einen weit gefächerten wie auch faszinierenden Überblick über die Selbstdarstellung von Staaten in den unterschiedlichsten Entwicklungsphasen und durch unterschiedlichste Mittel zu geben. Auch wenn manche Beiträge stark auf Architektur und Kunstgeschichte ausgerichtet sind, so kommt doch gelungen zum Ausdruck, mit welchen Bildern sich neue Staaten zu repräsentieren und legitimieren versuchen. Fazit: Der Sammelband ist für ein breites Publikum hoch interessant.

Sybille Reinke de Buitrago

\title{
Aktuelle Neuerscheinung
}

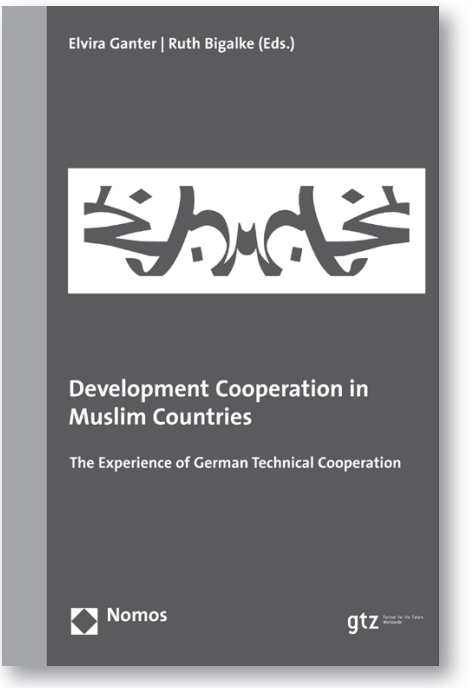

\author{
Development Cooperation in Muslim Countries \\ The Experience of German Technical Cooperation \\ Herausgegeben von Elvira Ganter und Ruth Bigalke \\ 2006, 114 S., brosch., 15,-€, ISBN 3-8329-2012-9
}

Das Werk analysiert die Grenzen und Potentiale einer Entwicklungszusammenarbeit mit muslimischen Ländern und leistet damit zugleich einen Beitrag zu einem dringend notwendigen Dialog mit den Partnerländern. Es wird dabei zudem deutlich, dass Entwicklungszusammenarbeit eine wichtige Komponente dieses Dialoges sein kann.

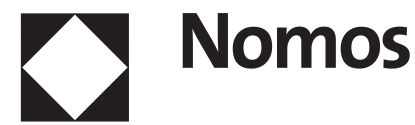

Bitte bestellen Sie bei Ihrer Buchhandlung oder bei: Nomos Verlagsgesellschaft 76520 Baden-Baden | www.nomos.de 